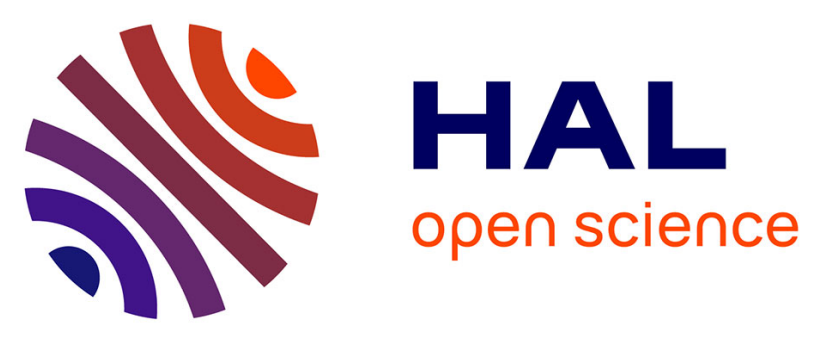

\title{
Continuous subcutaneous apomorphine infusion does not impair the dynamics of cognitive action control in mild to moderate Parkinson's disease
}

Joan Duprez, Jean-François Houvenaghel, Sophie Drapier, Manon Auffret, Dominique Drapier, Gabriel Robert, Marc Vérin, Paul Sauleau

\section{To cite this version:}

Joan Duprez, Jean-François Houvenaghel, Sophie Drapier, Manon Auffret, Dominique Drapier, et al. Continuous subcutaneous apomorphine infusion does not impair the dynamics of cognitive action control in mild to moderate Parkinson's disease. Journal of Neurology, 2018, 265 (3), pp.471-477. 10.1007/s00415-017-8721-7 . hal-01743548

HAL Id: hal-01743548

https://hal-univ-rennes1.archives-ouvertes.fr/hal-01743548

Submitted on 3 May 2018

HAL is a multi-disciplinary open access archive for the deposit and dissemination of scientific research documents, whether they are published or not. The documents may come from teaching and research institutions in France or abroad, or from public or private research centers.
L'archive ouverte pluridisciplinaire HAL, est destinée au dépôt et à la diffusion de documents scientifiques de niveau recherche, publiés ou non, émanant des établissements d'enseignement et de recherche français ou étrangers, des laboratoires publics ou privés. 
1Continuous subcutaneous apomorphine infusion does not impair the dynamics of cognitive action control in mild to 2moderate Parkinson's disease

3

4Joan Duprez ${ }^{\mathrm{a} *}$, Jean-François Houvenaghel ${ }^{\mathrm{a}, \mathrm{b}}$, Sophie Drapier ${ }^{\mathrm{a}, \mathrm{b}}$, Manon Auffret ${ }^{\mathrm{a}}$, Dominique Drapier ${ }^{\mathrm{a}, \mathrm{c}}$, Gabriel

5Robert ${ }^{\mathrm{a}, \mathrm{c}}$, Marc Vérin ${ }^{\mathrm{a}, \mathrm{b}} \&$ Paul Sauleau ${ }^{\mathrm{a}, \mathrm{d}}$

6

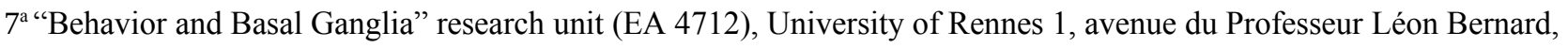
835000 Rennes, France

$9^{\mathrm{b}}$ Department of Neurology, Rennes University Hospital, rue Henri Le Guilloux, 35033 Rennes, France

$10^{c}$ Department of Psychiatry, Rennes University Hospital, 108 avenue du Général Leclerc, 35703 Rennes, France

$11^{\mathrm{d}}$ Department of Neurophysiology, Rennes University Hospital, rue Henri Le Guilloux, 35033 Rennes, France

12Jean-François Houvenaghel : Jeanfrancois.HOUVENAGHEL@chu-rennes.fr

13

14Sophie Drapier : Sophie.DRAPIER@chu-rennes.fr

15Manon Auffret : auffret.manon@gmail.com

16Dominique Drapier : d.drapier@ch-guillaumeregnier.fr

17Gabriel Robert : gabriel.hadrien.robert@gmail.com

18Marc Vérin : Marc.VERIN@chu-rennes.fr

19Paul Sauleau : Paul.SAULEAU@univ-rennes1.fr

20

21*Corresponding author: Joan Duprez, EA 4712 “Comportement et Noyaux Gris Centraux”, Université de Rennes 1 , 22Avenue du Professeur Léon Bernard, F-35043 Rennes Cedex, France

23Phone: +(33)2 992898 42, Fax: +(33)2 992841 32, Email: duprez.joan@gmail.com

24

25

26

27

\section{Acknowledgements}

29The authors would like to thank all the volunteers who took part in this study, as well as Elizabeth Wiles-Portier who 30revised the English style.

31 


\section{Conflict of interest statement}

2

3Dr Sophie Drapier received speech honorarium from Teva and Medtronic and served on scientific advisory

4boards for Aguettant and Britannia.

5Dr Marc Vérin has served on the Scientific Advisory Board for Aguettant and Orkyn and received speech honorarium

6from Teva and Medtronic.

7

8

9

10

11

12

13

14

15

16

17

18

19

20

21

22

23

24

25

26

27

28

29

30

31 
2Keywords: Cognitive action control; Parkinson's disease; CSAI; Activation-suppression; Simon task 3

\section{Abstract}

5Introduction: Continuous subcutaneous apomorphine infusion (CSAI) is increasingly used in Parkinson's disease (PD), 6notably in patients contraindicated for subthalamic deep brain stimulation. Although it has been suggested that CSAI is 7safe regarding cognition, few studies have actually investigated its effect, especially on cognitive control which is a 8crucial process for goal-directed behavior. More specifically, its impact on the dynamics of cognitive action control, as 9reflected by the activation and suppression of impulsive responses, has yet to be investigated, which is the objective of 10the present study.

11Methods: We compared cognitive action control between baseline (M0) and 6 months (M6) after the start of add-on

12CSAI by administering an oculomotor Simon task to 20 patients with mild to moderate PD. We used the

13activation-suppression model to determine whether CSAI had an effect on either the impulsive errors made in conflict 14 situations or the suppression of these responses.

15Results: We found no difference between M0 and M6 in the congruence effect regarding either reaction time or

16accuracy, indicating that overall conflict resolution was not influenced by CSAI. Furthermore, the rate of fast errors in 17the conflict situation and the last slope of the delta plots (reflecting the strength of impulsive response suppression) 18 were unaffected by the treatment. The $95 \%$ confidence intervals calculated for the treatment effect on both of these 19measures fell below the range of usual meaningful effects.

20Conclusion: We found no difference between M0 and M6, which strongly suggests that CSAI does not impair the 21dynamics of cognitive action control. 


\section{Introduction}

3

4Patients with Parkinson's disease (PD) whose motor fluctuations can no longer be satisfactorily controlled by oral 5 medication can be proposed continuous subcutaneous apomorphine infusion (CSAI). This treatment has repeatedly been Greported to have a beneficial effect, with reductions in motor fluctuations, as well as in nonmotor symptoms [1]. By 7contrast with previous reports [2], recent studies have suggested that the beneficial motor effect of CSAI comes without 8the deleterious cognitive side effects associated with other treatments such as deep brain stimulation of the subthalamic 9nucleus $[3,4,5]$. So far, however, few studies have examined the effect of CSAI on the cognitive functions thought to 10be affected by other antiparkinsonian treatments.

11

12One of the most important of these functions is cognitive control, as it allows us to adapt to our environment according 13to our intentions, ignoring any irrelevant information [6]. Among the different processes participating in an efficient 14 cognitive control, cognitive action control refers to the online control allowing to suppress unwanted and automatic 15action tendencies in favor of an intention-driven behavior [6]. This cognitive process is often assessed using 16experimental conflict tasks such as the Simon task [7]. This task requires participants to produce a "left" or "right" 17answer according to the color of a stimulus displayed on the left- or righthand side of a screen, regardless of its position. 18When the position and color of the stimulus are incongruent and indicate different responses, a conflict arises and the 19participant has to suppress the automatic response activated by the position of the stimulus. Incongruent situations lead 20 to more errors, and responding accurately has a cognitive cost expressed as an increase in reaction time (RT). This is 21commonly called the congruence effect.

22

23During the past decade, most studies investigating cognitive action control have focused on its dynamics, using the

24activation-suppression model and distributional analyses [8]. This model postulates that fast responses in incongruent 25situations are more error-prone, reflecting a process called impulsive action selection, while slow responses indicate a 26smaller congruence effect, reflecting the efficacy of a suppression mechanism. The conditional accuracy function 27(CAF) can be used to investigate impulsive action selection, as it represents accuracy as a function of congruence 28plotted against RT distribution, divided into several bins (usually 5-7). Lower accuracy for the first incongruent bin 29indicates stronger impulsive action selection. The suppression mechanism is shown by the delta plot representing the 30congruence effect (incongruent RT minus congruent RT for correct responses) as a function of RT distribution, as with 31the CAF. The steeper the negative slope between the two last bins, the greater the suppression (see [9] for a review). 
1The effect of PD and its treatment on these dynamics has been thoroughly studied in recent years. For instance, patients 2with PD have been shown to display stronger impulsive action selection and weaker suppression, compared with 3healthy controls $[10,11,12]$. Antiparkinsonian treatment also has an effect on these dynamics, as van Wouwe et al. [13] 4found that patients with PD displayed greater suppression when they were on their dopaminergic medication than when 5they were off medication. Furthermore, a study by Wylie et al. [14] revealed that dopamine agonists weaken the 6suppression of irrelevant activations, while the number of impulsive errors is unaffected. In this study, the dopamine 7agonist dose was correlated with the strength of suppression, with higher doses being associated with weaker 8suppression. Deep brain stimulation of the subthalamic nucleus impacts both impulsive action selection and 9suppression. Wylie et al. [15], for example, showed that patients undergoing active stimulation make more impulsive 10 errors than when they are off stimulation, but are more efficient at suppressing irrelevant activations, providing they 11take enough time to respond.

12

13Although some studies have suggested that CSAI has no deleterious cognitive effects, its potential effect on impulsive 14action selection and suppression in conflict situations has never been examined. CSAI does not seem to modify 15performances in standard neuropsychological assessments performed during routine clinical care [3, 4, 5, 16], but an 16effect on the dynamics of cognitive action control cannot be ruled out. Although the impact of classic oral agonists has 17already been studied [14], apomorphine differs from these dopaminergic agonists in terms of its pharmacodynamics and 18continuous administration modality [17]. The goal of the present study was to verify that add-on CSAI has no negative 19impact on cognitive action control and its dynamics. To this end, we used an oculomotor version of the Simon task that 20has proved to reveal subtle changes in cognitive action control in PD or healthy older participants [18, 10]. We 21compared the classic congruence effect, as well as the key features of impulsive action selection and suppression, 22measured before the introduction of CSAI and six months later in patients with mild to moderate PD.

\section{Methods}

25

26Table 1: Clinical data (mean \pm standard deviation) before (baseline) and after (M6) the start of CSAI. Statistical 27comparisons were carried out using the Wilcoxon paired test. Dyskinesia and fluctuation scores were calculated as the 28sum of items 32 to 35 and 36 to 39 (respectively) of the part IV of the UPDRS.

\begin{tabular}{|c|c|c|c|c|c|}
\hline & $\mathrm{N}$ & Baseline & M6 & p-value & V statistic \\
\hline Sample size & & 20 & 20 & & \\
\hline Age (years) & $20 / 20$ & $58.9 \pm 10.5$ & - & & \\
\hline Education (years) & $20 / 20$ & $19.2 \pm 8.7$ & - & & \\
\hline Sex (M:F) & $20 / 20$ & $9: 11$ & - & & \\
\hline Disease duration & $20 / 20$ & $11.7 \pm 4.8$ & - & & \\
\hline
\end{tabular}




\begin{tabular}{|c|c|c|c|c|c|}
\hline Hoehn and Yahr rating-ON & $18 / 20$ & $1,1 \pm 1$ & $0.8 \pm 0.8$ & 0.32 & $\mathrm{~V}=37.5$ \\
\hline Hoehn and Yahr rating-OFF & $18 / 20$ & $2.1 \pm 0.8$ & $2.1 \pm 0.6$ & 0.65 & $\mathrm{~V}=27.5$ \\
\hline Schwab \& England rating - ON & $18 / 20$ & $91.7 \pm 7.1$ & $92.3 \pm 9$ & 0.86 & $\mathrm{~V}=12.5$ \\
\hline Schwab \& England rating - OFF & $18 / 20$ & $71.1 \pm 16$ & $75.9 \pm 17$ & 0.5 & $\mathrm{~V}=48$ \\
\hline UPDRS II On med & $20 / 20$ & $4.4 \pm 3.2$ & $4.8 \pm 3.1$ & 0.98 & $\mathrm{~V}=67$ \\
\hline UPDRS II Off med & $20 / 20$ & $15.5 \pm 4.7$ & $13.7 \pm 6$ & 0.3 & $\mathrm{~V}=96.5$ \\
\hline UPDRS III On med & $20 / 20$ & $13.2 \pm 9.2$ & $13 \pm 8.2$ & 0.9 & $\mathrm{~V}=66.5$ \\
\hline UPDRS III Off med & $19 / 20$ & $35.3 \pm 12.19$ & $36.7 \pm 10$ & 0.5 & $\mathrm{~V}=48$ \\
\hline UPDRS IV & $18 / 20$ & $6.1 \pm 2.2$ & $5.1 \pm 2.3$ & 0.2 & $\mathrm{~V}=73$ \\
\hline Dyskinesia score (Items 32-35) & $18 / 20$ & $2.1 \pm 1.5$ & $2.1 \pm 1.6$ & 0.7 & $\mathrm{~V}=50.5$ \\
\hline Fluctuation score (Items 36-39) & $18 / 20$ & $\mathbf{3 . 2} \pm \mathbf{1 . 2}$ & $\mathbf{2 . 1} \pm \mathbf{1 . 1}$ & $\mathbf{0 . 0 2}$ & $\mathrm{V}=59$ \\
\hline LEDD total (mg/day) & $20 / 20$ & $\mathbf{1 0 8 9 . 2} \pm \mathbf{2 6 5 . 2}$ & $\mathbf{1 4 9 9 . 8} \pm \mathbf{3 4 3 . 7}$ & $\mathbf{0 . 0 0 1}$ & $\mathrm{V}=13$ \\
\hline Levodopa & $20 / 20$ & $\mathbf{8 3 7 . 6} \pm \mathbf{2 8 8 . 5}$ & $\mathbf{4 7 9 . 4} \pm \mathbf{2 2 6 . 6}$ & $\mathbf{0 . 0 0 1}$ & $\mathrm{V}=145$ \\
\hline Agonist & $20 / 20$ & $245 \pm 125.6$ & $195.3 \pm 121$ & 0.05 & $\mathrm{~V}=64$ \\
\hline Apomorphine & $20 / 20$ & - & $826.9 \pm 207.5$ & & \\
\hline LEDD total oral med (mg/day) & $20 / 20$ & $\mathbf{1 0 8 2 . 6} \pm \mathbf{2 5 2 . 3}$ & $\mathbf{6 7 4 . 7} \pm \mathbf{2 4 4 . 5}$ & $\mathbf{0 . 0 0 0 5}$ & $\mathrm{V}=150$ \\
\hline MDRS & $20 / 20$ & $138 \pm 4.5$ & $137.7 \pm 5.2$ & 0.7 & $\mathrm{~V}=75.5$ \\
\hline
\end{tabular}

1 Note. UPDRS = Unified Parkinson's Disease Rating Scale; LEDD = levodopa equivalent daily dose; MDRS = Mattis

Dementia Rating Scale

\subsection{Patients}

4We recruited 20 patients with idiopathic PD from the Neurology Department of Rennes University Hospital (France)

5 for inclusion in this study (Table 1). They had mild to moderate disease severity (Hoehn and Yahr stages I-III in the on-

6medication state [19]) and had been hospitalized in order to introduce apomorphine hydrochloride treatment

7(Apokinon $\Re$; Aguettant, Lyon, France) using an infusion pump (Microjet Crono PAR; Cane Medical Technology, Italy)

8as part of their routine clinical care. The CSAI treatment had been proposed to these patients due to motor fluctuations

9occurring despite an optimized oral treatment. Before CSAI, these patients displayed an averaged fluctuation score

10 (calculated as the sum of items 36 to 39 of the UPDRS IV) of $3.2 \pm 1.2$ and an average off-state score of $1.15 \pm 0.5$.

11This score represents a proportion of 26 to $50 \%$ of off-state during the day. CSAI has been reported to reduce these off-

12 state periods known to have a strong negative impact on patients' daily life [20, 21]. Patients were free of severe

13cognitive deterioration (Mattis Dementia Rating Scale score > 130; [22]), and had no other neurological or psychiatric

14pathologies. The severity of their motor symptoms was assessed using the third part of the Unified Parkinson's Disease

15Rating Scale (UPDRS). All patients were assessed under their usual medication at baseline (before the introduction of

16CSAI) and after 6 months of apomorphine treatment (M6; mean time since baseline: $6 \pm 0.3$ months). At baseline,

17medication included both dopamine agonists and levodopa therapy in 19 patients, and levodopa alone in one patient.

18The study was approved by the ethics committee of Rennes University Hospital and conducted in accordance with the

19Declaration of Helsinki and current French legislation (Huriet Act). All the patients gave their informed consent to take

20part in the study.

21

222.2. CSAI treatment 
1CSAI was used during the day for a mean duration of $13.6 \pm 4$ hours (range: 6-20) at a mean hourly rate of $4.9 \pm 0.8 \mathrm{mg}$ 2(range: $3.8-7$ ) and a mean bolus number of $2.6 \pm 1.5$ per day (range: $0-5$ ) with a mean dose of $3.4 \pm 1.3 \mathrm{mg}$ (range: $0-5$ ) 3per bolus.

\section{4}

\subsection{Task design and procedure}

6We used an oculomotor version of the Simon task in which participants were instructed to make a left or right eye 7movement according to the color (blue or yellow) of a stimulus, while ignoring its location (left or right), as fast and 8accurately as possible. Participants performed a total of 300 trials, divided into five blocks with self-paced breaks 9between these blocks. Two types of situation happened pseudorandomly with an equal probability: a congruent one 10 where the location of the stimulus corresponded to the response side indicated by its color; and an incongruent one 11where the location and color of the stimulus indicated opposite responses. Color mapping was pseudorandomly 12assigned to each patient, and the reverse mapping was used at M6. Eye movements were recorded using an EyeBrain 13T2 ${ }^{\circledR}$ head-mounted eyetracker (e(ye)BRAIN, Ivry-sur-Seine, France) with a $300 \mathrm{~Hz}$ sampling rate and an angular 14resolution of $0.5^{\circ}$. For more details, see [18].

15

162.4. Analysis

17

18All data analyses were performed using R@ software (Version 3.1.0). The first eye movement after stimulus 19 presentation was deemed to be the participant's response, and was considered correct if it corresponded to the side 20 indicated by the color of the stimulus. Eye movements with an amplitude below $2^{\circ}$ and a latency below $100 \mathrm{~ms}$ or 21above $1000 \mathrm{~ms}$ were removed (for more details, see [18]). Outlier latencies of more than three standard deviations from 22 mean RT were also discarded. Overall, $2.03 \%$ of the data were removed. For each participant, we calculated the mean 23RT for correct trials and mean accuracy for each congruence condition, to evaluate the congruence effect. We also used 24distributional analyses to assess impulsive action selection and suppression [8]. We computed CAFs and delta plots 25showing accuracy and the congruence effect (incongruent RT minus congruent RT of correct trials) as a function of RT 26distribution divided into five bins (see [18]). The first bin of each CAF in the incongruent situation was used to assess 27impulsive action selection, and the value of the final slope of each delta plot was used to assess suppression [9].

28

29As accuracy was not normally distributed, it was arcsine transformed prior to the statistical analysis. The congruence 30effect, accuracy for the first bin of the incongruent CAF, and last slope value of the delta plot were compared between 31baseline and M6 using repeated-measures analyses of variance (ANOVAs). For each test, we checked the assumptions 
1of normality and homoscedasticity of the residuals. Clinical variables were compared using paired Wilcoxon signed2rank tests. The significance threshold was set at $p=0.05$.

3

\section{Results}

5

\subsection{Clinical effect of CSAI}

7

8We found a significant decrease in motor fluctuations, as assessed with the sum of Items 36-39 of the UPDRS-IV at 9M6, compared with baseline (Table $1 ; \mathrm{V}=59, p=0.02$ ). Oral treatment was significantly reduced at M6, with a 10significant decrease in the levodopa equivalent daily dose (LEDD; $\mathrm{V}=145, p=0.001)$, as well as in the total oral 11 medication daily dose $(\mathrm{V}=150, p=0.0005)$. The oral agonist dose tended to decrease $(\mathrm{V}=64, p=0.05)$ whereas 12overall LEDD increased, owing to the introduction of apomorphine treatment $(\mathrm{V}=13, p=0.001)$.

13

143.2. Congruence effect

15

16We found no difference between baseline and M6 in overall RT, suggesting that patients responded at the same speed 17 before and after the introduction of $\operatorname{CSAI}(F(1,19)<1, p=0.4)$. A strong congruence effect was found, with a 18significantly shorter RT in the congruent situation than in the incongruent one reflecting the cognitive cost of conflict 19resolution (Fig. $1 ; F(1,19)=34.9, p<0.0001$ ). No difference was found in the size of the congruence effect between 20baseline and M6, as revealed by the absence of a significant interaction effect (mean congruence effect $=89 \pm 62 \mathrm{~ms}$ at 21 baseline and $111 \pm 72 \mathrm{~ms}$ at $\mathrm{M} 6 ; F(1,18)<1, p=0.5)$. This result suggests that the cost of conflict resolution was 22unaffected by the introduction of CSAI.

23

24Overall accuracy did not differ between baseline and M6, suggesting that CSAI had no impact on patients' precision $25(F(1,19)<1, p=0.9)$. As with RT, we found a strong congruence effect, with a significant decrease in accuracy in the 26incongruent situation, compared with the congruent one (Fig. $1 ; F(1,19)=138.2, p<0.0001)$. The size of the 27congruence effect on accuracy was unaffected by CSAI, as suggested by the absence of a significant interaction effect 28 (mean congruence effect $=39 \% \pm 28$ at baseline and $46 \% \pm 28$ at M6; $F(1,18)=1.4, p=0.24)$. 

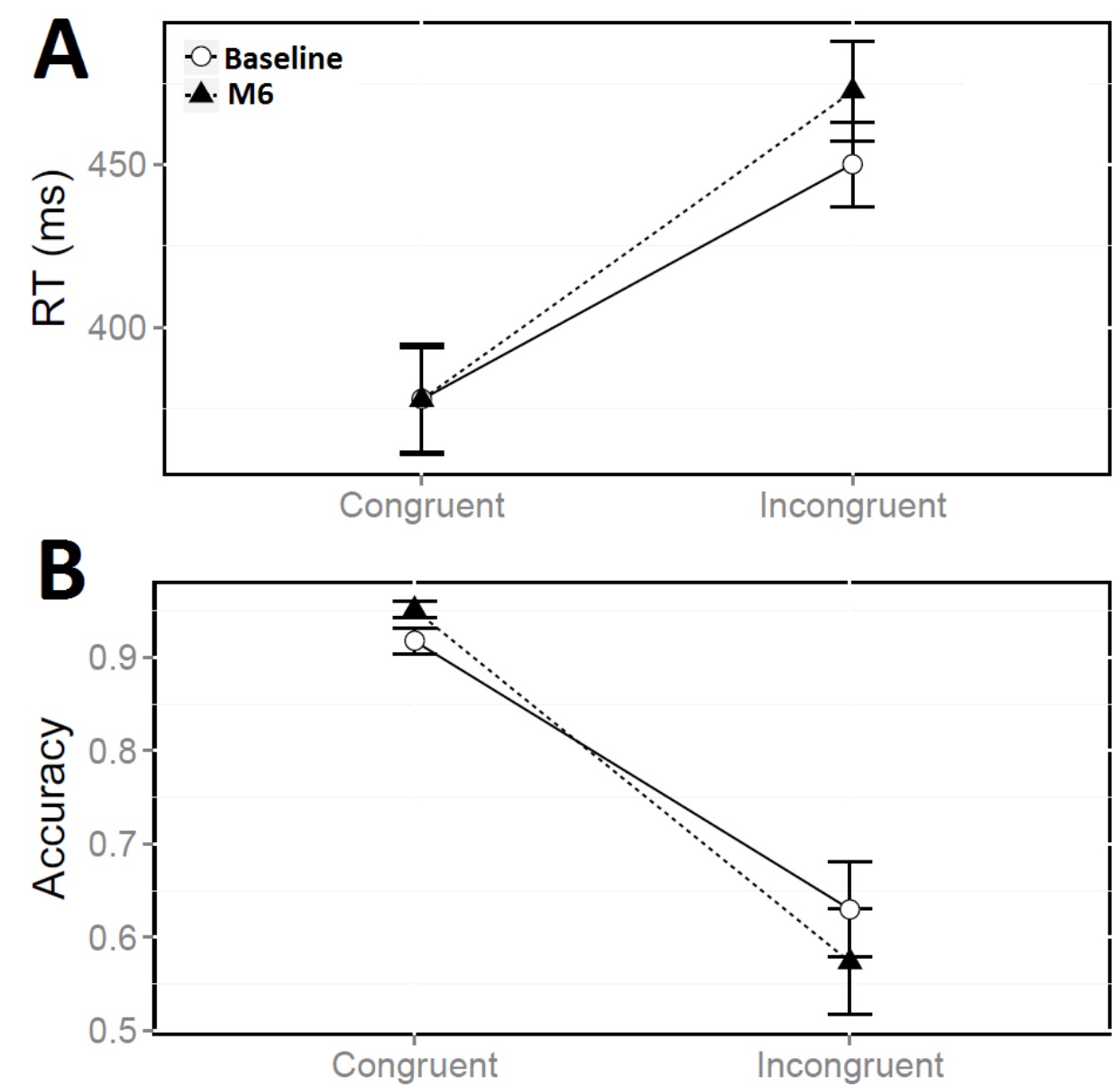

2 Figure 1: Mean RT (A) and accuracy (B) according to congruence and treatment phase. Error bars represent the 3 standard error of the mean.

53.3. Impulsive action selection

6

7We found no difference between baseline and M6 in accuracy for the first bin of the incongruent situation (Fig. 2; $F(1$, $819)=0.19, p=0.66$ ). This suggests that patients made just as many impulsive errors 6 months after the introduction of 9CSAI as they did before. Impulsive action selection is regarded as a key component of the dynamics of cognitive action 10 control, and was our primary focus in this study, along with selective suppression. As the absence of an effect cannot be 11ruled out solely on the basis of the absence of a significant difference, we computed the $95 \%$ confidence interval of the 12effect between baseline and M6 for impulsive action selection. The mean difference between baseline and M6 was -0.03 13(95\% CI [-0.09, 0.04]), indicating a negligible decrease in accuracy between baseline and M6. Group differences 14reported for PD in the Simon task literature are about $0.1[11,15,23]$. As the $95 \%$ confidence interval for the 15differences in our study fell below what is usually reported as a meaningful difference, we can safely assume that there 16were no differences between baseline and M6 on impulsive action selection in our study. 


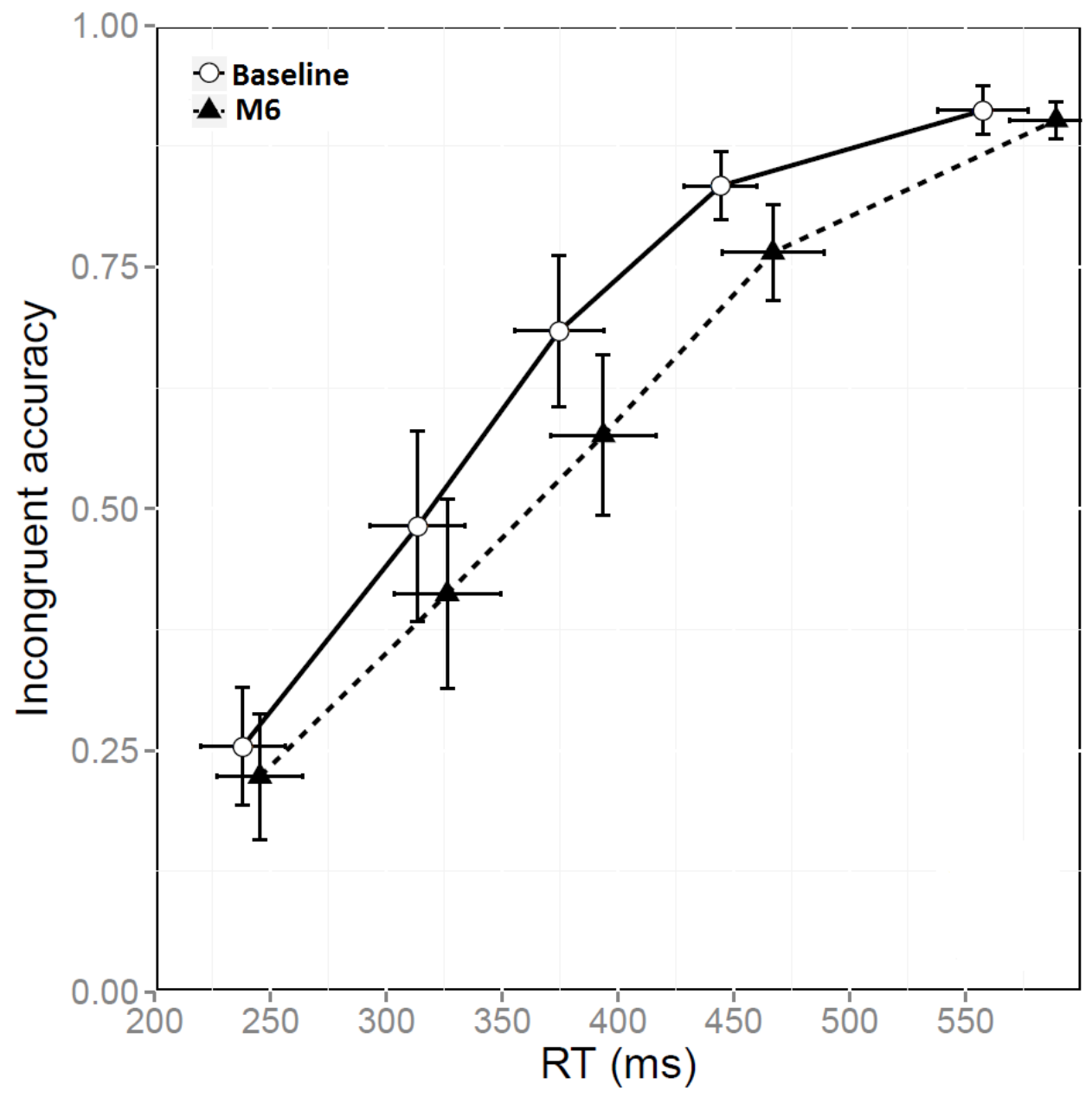

2Figure 2: CAF for the incongruent situation, plotted as a function of RT distribution divided into five bins. Accuracy for

3 the first bin indicates the strength of impulsive action selection. Error bars represent the standard error of the mean.

4

53.4. Suppression of the irrelevant activation

6

7There was no significant difference between baseline and M6 in the slope value of the final bin of the delta plot (Fig. 3; $8 F(1,19)<1, p=0.88)$. This suggests that CSAI did not weaken the suppression process, as it remained at its baseline 9level. As for impulsive action selection, we computed the $95 \%$ confidence interval for the mean difference between 10baseline and M6 to see whether there was a potentially meaningful effect in our data despite the absence of a significant 11difference. The mean difference between baseline and M6 was -0.01 (95\% CI [-0.18, 0.15]). In the literature on conflict 12 control in PD, significant differences are usually about $0.2[15,14,13]$, which is outside the range of the $95 \%$ 13confidence interval for our data. This strongly supports the absence of an effect between baseline and M6 in our study. 


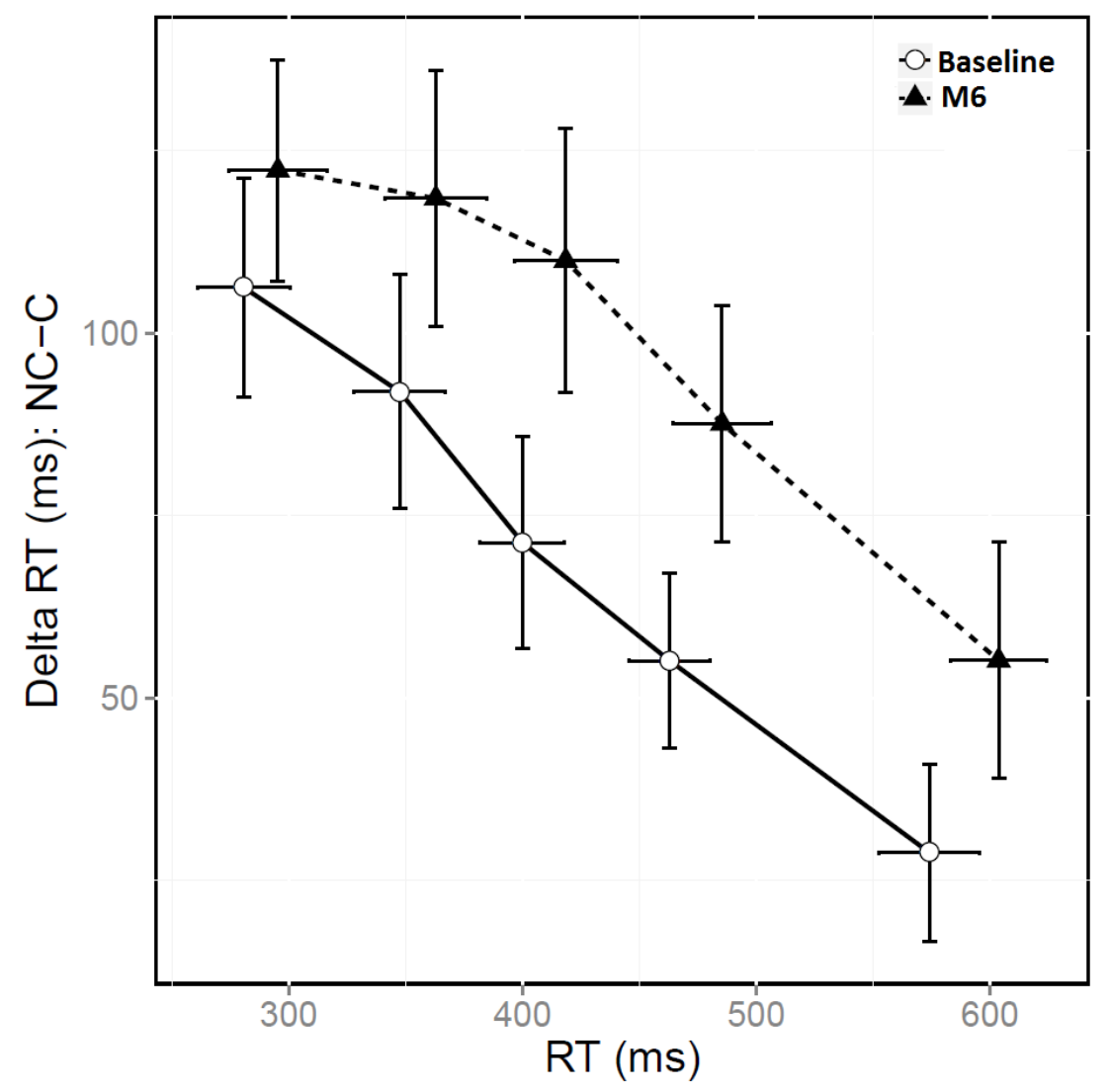

2Figure 3: Delta plots showing the congruence effect as a function of RT distribution, divided into five bins. The value of 3 the final slope indicates the strength of selective suppression. Error bars represent the standard error of the mean.

4

\section{Discussion}

6

7In the current study, we wanted to check that add-on CSAI has no impact on a key cognitive process (i.e., cognitive 8action control), and more specifically on the dynamics of this process, as reflected by impulsive action selection and 9suppression.

10

11Regarding motor improvement, motor fluctuations decreased and oral medication was reduced accordingly, in line with 12the literature $[3,24,20]$. Regarding cognitive action control, as expected, our results revealed no difference in the 13congruence effect between baseline and 6 months after the introduction of CSAI. In other words, the cost of conflict 14resolution was unaffected by the introduction of apomorphine. The primary focus of our study was the effect of CSAI 15on impulsive action selection (reflected by accuracy for the first CAF bin in the incongruent situation) and suppression 16(reflected by the last delta plot slope value). We found that patients displayed similar accuracy at baseline and M6 for 17 fast incongruent responses, and exerted equally strong suppression of automatic activations. The $95 \%$ confidence 18intervals for the differences between baseline and M6 on these two measures were not within the range of the 
1meaningful effects that are usually reported in the literature using the Simon task, arguing in favor of an absence of 2effect. Thus, our results strongly suggest that CSAI has no impact on impulsive action selection and suppression in 3patients with mild to moderate PD, in accordance with previous reports of the absence of neuropsychological 4impairment after CSAI [3, 4, 5].

5

6The absence of a deterioration in cognitive action control may arise from two aspects of CSAI treatment. First, the 7overall action of the apomorphine molecule may differ from that of other dopamine agonists. Apomorphine is a 8nonselective dopamine agonist that can interact with both D1-like and D2-like receptors [17], unlike most other 9antiparkinsonian dopamine agonists, which mainly bind to D2-like receptors. Wylie et al. [14] reported that weaker 10suppression of automatic activation correlated with higher doses of dopamine agonists (agonists binding to D2-like 11receptors). As apomorphine differs from the usual agonists, it may well have a different effect on cognitive action 12control. Another, nonexclusive, hypothesis is that the continuous nature of CSAI treatment, as opposed to the pulsatile 13doses of standard oral medication, results in greater physiological dopaminergic stimulation without any fluctuation in 14dose effects. This may stem either from the continuous administration modality of the apomorphine itself or from the 15 overall reduction in oral treatment. In turn, this may maintain a certain level of cognitive performance, such as cognitive 16action control in the current study. Previous studies investigating the cognitive effect of antiparkinsonian treatments 17 compared the performances of patients between their best (on medication) and worst (off medication) motor conditions. 18It is therefore difficult to compare the results of other studies with our own, and further investigations are needed to test 19the hypothesis that continuous versus pulsatile treatment has differential effects on cognitive action control.

\section{0}

21Our study had several limitations. The patients in our group were free from cognitive deterioration at baseline and their 22disease was generally less advanced than that of participants included in previous studies [3, 4, 5]. This raises the 23question of a potential differential effect of CSAI on cognitive action control in patients with or without cognitive 24impairment. Thus, although we can hypothesize that CSAI is safe regarding cognitive action control in patients with 25 mild to moderate disease severity, the generalizability of its effect to patients at a more advanced stage of the disease

26needs to be investigated further. However, it should be noted that the recent study of Borgemeester et al. [16] has shown 27that CSAI had no negative impact on executive functioning in PD patients with cognitive dysfunction. Another point is 28that the absence of an effect on cognitive action control in our study has to be offset against the size of the patient 29sample and the specificity of the task. Confirmation of our results requires similar studies using other cognitive tasks 30and a larger group of patients. Lastly, it would be very interesting to directly compare the effects of apomorphine and 31other dopamine agonists on cognitive action control. 


\section{Conclusion}

2The present study was the first to investigate the effect of CSAI on the dynamics of cognitive action control. Our results 3strongly suggest that CSAI has no negative impact on impulsive action selection and suppression in patients with mild 4to moderate PD. These results are important, as the nonmotor and cognitive effects of antiparkinsonian treatments have 5a major impact on patients' quality of life (see [1] for a review). Although further studies are needed to confirm the 6absence of cognitive deterioration with CSAI, our results argue strongly in favor of its safety for cognitive action 7control.

\section{Ethical standards}

10

11This study was approved by the ethics committee of Rennes University Hospital and conducted in accordance with the 12Declaration of Helsinki and current French legislation (Huriet Act).

13All the patients gave their informed consent to take part in the study.

14

\section{Source of funding}

16

17This research did not receive any specific grant from funding agencies in the public, commercial, or not-for-profit 18sectors.

19

\section{References}

21

221. Rosa-Grilo M, Qamar MA, Evans A, Chaudhuri KR (2016) The efficacy of apomorphine - A non-motor 23perspective. Parkinsonism Relat Disord 33:S28-S35. doi: 10.1016/j.parkreldis.2016.11.020

24

252. Růzicka E, Roth J, Spacková N, et al (1994) Apomorphine induced cognitive changes in Parkinson's disease. J 26Neurol Neurosurg Psychiatry 57:998-1001.

27

283. Alegret M, Valldeoriola F, Martí M, et al (2004) Comparative cognitive effects of bilateral subthalamic 29stimulation and subcutaneous continuous infusion of apomorphine in Parkinson's disease. Mov Disord 19:1463-1469. 
14. De Gaspari D, Siri C, Landi A, et al (2006) Clinical and neuropsychological follow up at 12 months in patients 2 with complicated Parkinson's disease treated with subcutaneous apomorphine infusion or deep brain stimulation of the 3subthalamic nucleus. J Neurol Neurosurg Psychiatry 77:450-453.

\section{4}

55. Drapier S, Gillioz A-S, Leray E, et al (2012) Apomorphine infusion in advanced Parkinson's patients with 6subthalamic stimulation contraindications. Parkinsonism Relat Disord 18:40-44.

7

86. Richard Ridderinkhof K, Forstmann BU, Wylie SA, et al (2011) Neurocognitive mechanisms of action control: 9resisting the call of the Sirens. Wiley Interdiscip Rev Cogn Sci 2:174-192.

10

117. Simon JR (1969) Reactions toward the source of stimulation. J Exp Psychol 81:174.

12

138. Ridderinkhof KR, others (2002) Activation and suppression in conflict tasks: Empirical clarification through 14distributional analyses.

159. van den Wildenberg WPM, Wylie SA, Forstmann BU, et al (2010) To head or to heed? Beyond the surface of 16selective action inhibition: a review. Front Hum Neurosci 4:222. doi: 10.3389/fnhum.2010.00222

17

1810. Duprez J, Houvenaghel J-F, Argaud S, et al (2017) Impulsive oculomotor action selection in Parkinson's 19disease. Neuropsychologia 95:250-258. doi: 10.1016/j.neuropsychologia.2016.12.027

20

2111. Wylie SA, van den Wildenberg WPM, Ridderinkhof KR, et al (2009) The effect of Parkinson's disease on 22interference control during action selection. Neuropsychologia 47:145-157.

23

2412. Wylie SA, Ridderinkhof KR, Bashore TR, van den Wildenberg WPM (2010) The effect of Parkinson's disease 25on the dynamics of on-line and proactive cognitive control during action selection. J Cogn Neurosci 22:2058-2073. doi: 2610.1162/jocn.2009.21326

27

2813. van Wouwe NC, Kanoff KE, Claassen DO, et al (2016) Dissociable Effects of Dopamine on the Initial Capture 29and the Reactive Inhibition of Impulsive Actions 0in Parkinson's Disease. J. Cogn. Neurosci.

30

3114. Wylie SA, Claassen DO, Huizenga HM, et al (2012) Dopamine agonists and the suppression of impulsive

32motor actions in Parkinson disease. J Cogn Neurosci 24:1709-1724. 
215. Wylie SA, Ridderinkhof KR, Elias WJ, et al (2010) Subthalamic nucleus stimulation influences expression and 3suppression of impulsive behaviour in Parkinson's disease. Brain J Neurol 133:3611-3624. doi: 10.1093/brain/awq239

4

516. Borgemeester R W K, van Laar T (2017). Continuous subcutaneous apomorphine infusion in Parkinson's 6disease patients with cognitive dysfunction : A retrospective long-term follow-up study. Parkinsonism Relat Disord. 7doi: 10.1016/j.parkreldis.2017.09.025.

8

917. Ribarič S (2012) The pharmacological properties and therapeutic use of apomorphine. Mol Basel Switz 1017:5289-5309. doi: 10.3390/molecules17055289

11

1218. Duprez J, Houvenaghel J-F, Argaud S, et al (2016) Enhanced Impulsive Action Selection in Middle-Aged 13Adults-Insights From an Oculomotor Simon Task. Front Aging Neurosci 8:251. doi: 10.3389/fnagi.2016.00251

14

1519. Hoehn MM, Yahr MD (1967) Parkinsonism onset, progression, and mortality. Neurology 17:427-427. doi: 1610.1212/WNL.17.5.427

17

1820. Drapier S, Eusebio A, Degos B, et al (2016) Quality of life in Parkinson's disease improved by apomorphine 19pump: the OPTIPUMP cohort study. J Neurol 263:1111-1119. doi: 10.1007/s00415-016-8106-3

20

2121. Martinez-Martin P, Reddy P, Antonini A, Henriksen T, Katzenschlager R, Odin P, Todorova A, Naidu Y, Tluk 22S, Chandiramani C, Martin A, Chaudhuri K R (2011) Chronic subcutaneous infusion therapy with apomorphine in 23advanced Parkinson's disease compared to conventional therapy: a real life study of non motor effect. J Park Dis 1:19724203. doi : 10.3233/JPD-2011-11037.

25

2622. Mattis S (1988) Dementia rating scale professional manual. Psychological Assessment Resources, Odessa 2723. Wylie SA, van den Wildenberg W, Ridderinkhof KR, et al (2012) Differential susceptibility to motor 28impulsivity among functional subtypes of Parkinson's disease. J Neurol Neurosurg Psychiatry 83:1149-1154. doi: 2910.1136/jnnp-2012-303056

30

3124. Katzenschlager R, Poewe W, Rascol O, Trenkwalder C, Deuschl G, Chaudhuri R, Henriksen T, van Laar T, 32Spivey K, Vel S, Lees A (2017) Double-blind, randomized, placebo-controlled, Phase III study (TOLEDO) to evaluate 
1the efficacy of apomorphine subcutaneous infusion in reducing OFF time in Parkinson's disease patients with motor 2fluctuations not well controlled on optimized conventional treatment [abstract]. Mov Disord. 32 (suppl 2)

3 